\title{
A DECENTRALIZED CRYPTOGRAPHIC BLOCKCHAIN APPROACH FOR HEALTH INFORMATION SYSTEM
}

\author{
Dr.J.Chenni Kumaran, Antony Eric Machado ${ }^{2}$, Dharanidharan R ${ }^{3,}$ Rishi Johnson Bose ${ }^{4}$. \\ ${ }^{1}$ Associate Professor, ${ }^{2,3,4} \mathrm{UG}$ Scholar Department of Information Technology, \\ Panimalar Institute of Technology, Chennai. \\ Department of Information Technology, Panimalar Institute of Technology, Chennai.
}

\begin{abstract}
Blockchain had been an exciting studies vicinity for a long term and the advantages it gives had been utilized by some of diverse industries. Similarly, the healthcare zone stands to advantage immensely from the blockchain generation because of protection, privacy, confidentiality and decentralization. Nevertheless, the Electronic Health Record (EHR) structures face issues concerning records protection, integrity and management. In this paper, we speak how the blockchain generation may be used to convert the EHR structures and might be an answer of those issues. We gift a framework that would be used for the implementation of blockchain generation in healthcare zone for EHR. The goal of our proposed framework is first off to put into effect blockchain generation for EHR and secondly to offer stable garage of digital facts via way of means of defining granular get entry to guidelines for the customers of the proposed framework. Moreover, this framework additionally discusses the scalability trouble confronted via way of means of the blockchain generation in preferred through use of off-chain garage of the facts. This framework gives the EHR device with the advantages of getting a scalable, stable and imperative blockchain-primarily based totally answer with use of sha256 hashing algorithm.
\end{abstract}

Keywords: Blockchain, Healthcare, EHR System, SHA 256, Decentralized.

\section{INTRODUCTION}

Many researchers have attempted to use synthetic intelligence into the sector of healthcare to help medical doctors withinside the prognosis and remedy of sicknesses. Besides, a few clinical choice-making structures primarily based totally on processing to go looking remedy answers in a big range of unstructured literatures and affected person facts including the patients' clinical history, symptoms, and laboratory exams to offer choice helps for medical doctors. Although clever healthcare structures have made surprising development in current years, conditions in realistic packages are nevertheless some distance from ideal. On the only hand, regardless of maximum medical doctors have an excellent know-how in their personal fields, an increasing number of sicknesses require cross-border clinical know-how of professionals from unique backgrounds, as a consequence it will become important to have them paintings collectively the use of technological means. On the alternative hand, because of the local and man or woman differentiations amongst patients, the needs for specific clinical care, personalized prognosis, and remedy are increasing, which makes the correct affected person picturing ever extra crucial than before.

\section{SYSTEM DESCRIPTION}

\section{EXISTING SYSTEM}

In existence device, a framework of parallel healthcare structures (PHSs) primarily based totally at the synthetic structures computational experiments and parallel execution (ACP) technique is proposed on this paper. PHS makes use of synthetic healthcare structures to version and constitute patients' conditions, prognosis, and remedy process, then applies computational experiments to research and compare diverse healing regimens, and implements parallel execution for healthcare processes.

\section{DISADVANTAGE}

1. It makes use of too easy algebraic structure.

2. Every block is usually encrypted withinside the identical manner.

3. Hard to put into effect with software program.

4. AES in counter mode is complicated to put into effect in software program taking each overall performance and protection into considerations.

\section{PROPOSED SYSTEM}

1. SHA-256 is a one-manner characteristic that converts a textual content of any period right into a string of 256 bits. This is called a hashing characteristic. In this case, it's miles a cryptographically stable hashing characteristic, in that understanding the output tells you little or no approximately the enter. It is a changed model of SHA1, which in flip is a changed SHA0. All 3 at the moment are broken, to a few extent.

2. Note 1: All variables are 32 bit unsigned integers and addition is calculated modulo 232

3. Note 2: For every spherical, there may be one spherical consistent $\mathrm{k}[\mathrm{i}]$ and one access withinside the message agenda array $\mathrm{w}[\mathrm{i}], 0 \leq \mathrm{i} \leq 63$

4. Note 3: The compression characteristic makes use of eight operating variables, a via $h$ 
5. Note 4: Big-endian conference is used while expressing the constants on this pseudocode, and while parsing message block records from bytes to words, for example, the primary phrase of the enter message "abc" after padding is 0x61626380.

\section{Advantage}

1.The leader benefit that SHA-256 has extra efficient.

2.It is rapid to compute, proof against pre-photograph and secondpreimage attacks, and is collision resistant.

\section{SYSTEM DESIGN}

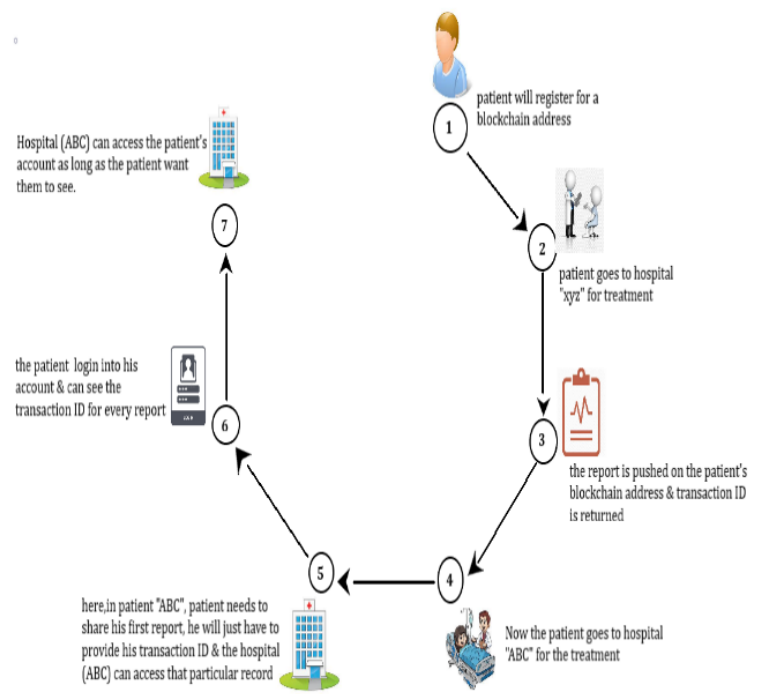

Figure 1: Architecture Diagram

\section{RESULTS AND DISCUSSION}

\section{A. Running the Program:}

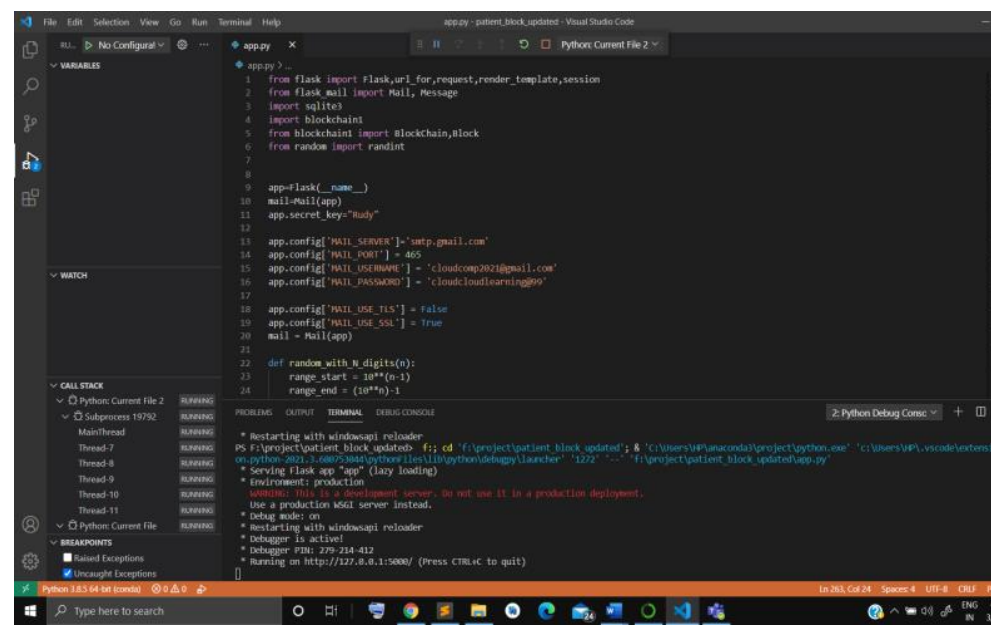

Figure 2: Running the Program

\section{B. Patient Login:}

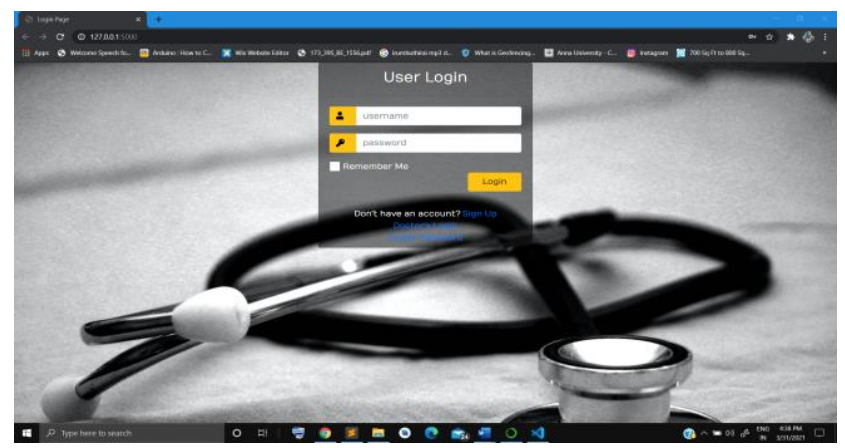

Figure 3: Patient Login

C. Patient Signup Page:

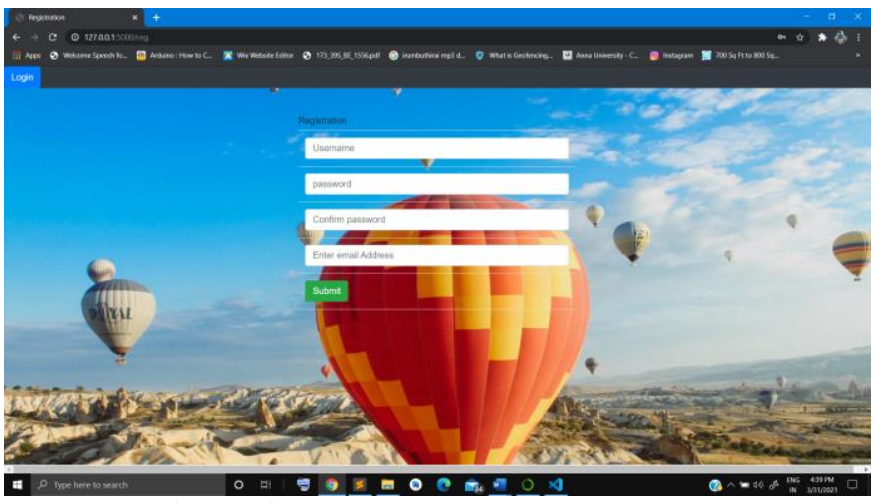

Figure 4: Patient Signup page.

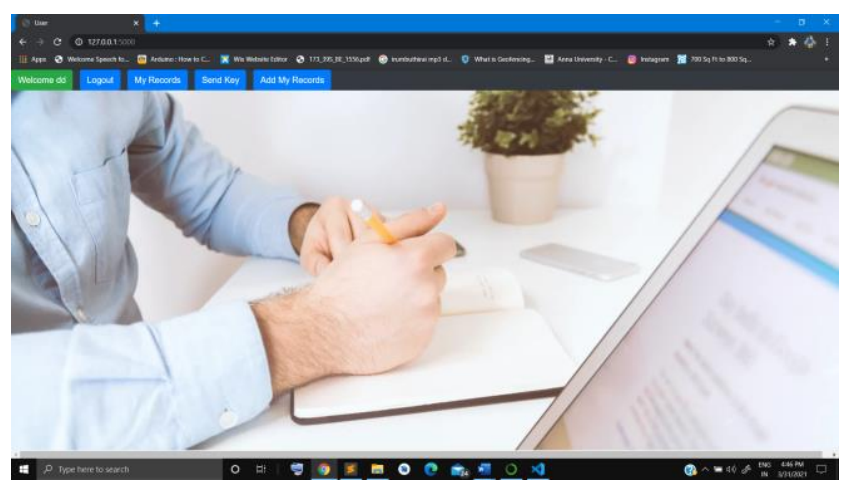

Figure 5 : Patient page 
E. Adding the affected person facts:
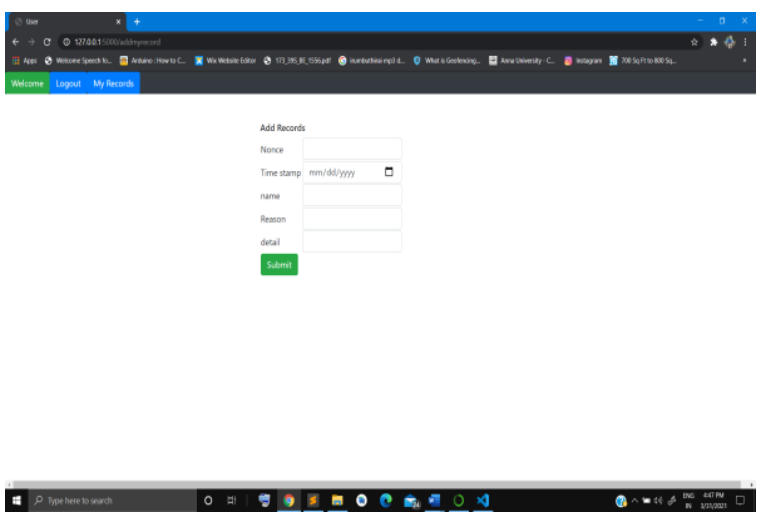

Figure 6: Adding the affected person facts.

F. Sending Key to Doctor:

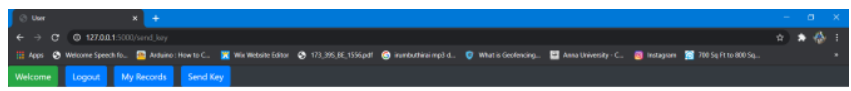

5 stomit
H. Enter OTP Sent To Doctor's Mail id:

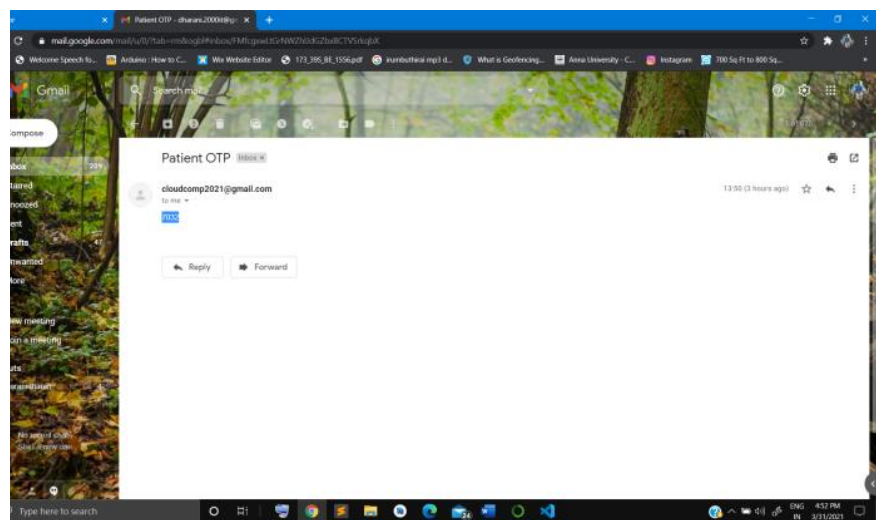

Figure 9: Enter OTP despatched to Doctor's mail id

I. ENTER OTP:

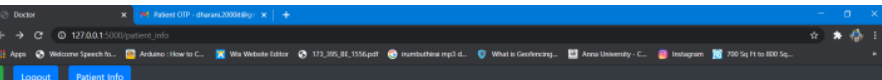

sibmit

\section{Figure 7: Sending key to doctor.}

G. Doctor Login Page:

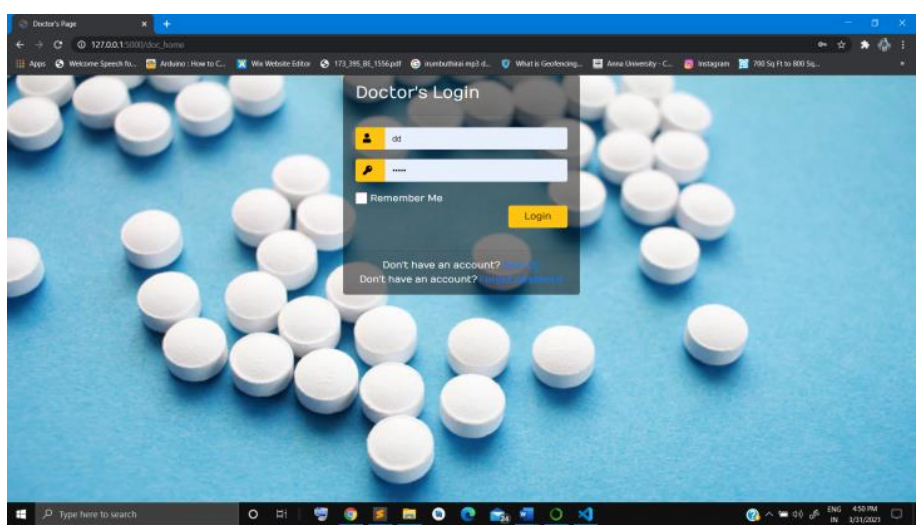

Figure 8: Doctor Login Page.
J. UPDATE patient info:

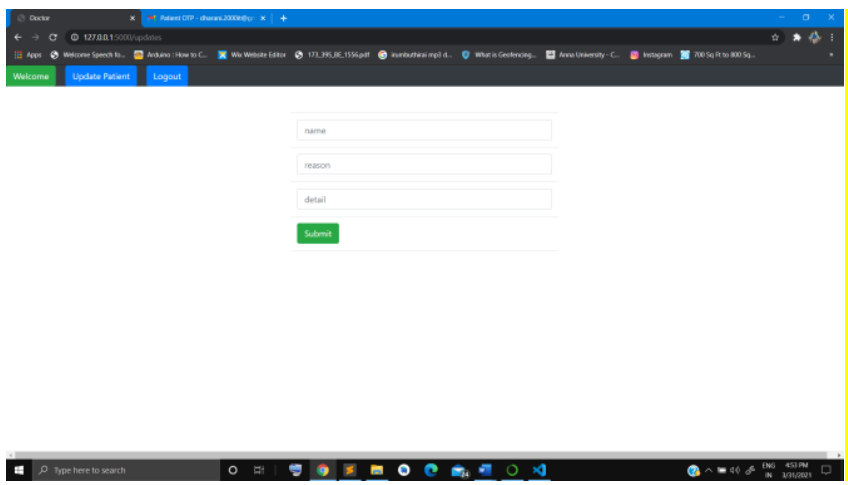

Figure 11: UPDATE affected person info: 


\section{CONCLUSION}

Blockchain enables the organization of check, characterization, and data sharing while in the meantime giving information related to assurance, helpful resource-saving and empowering for the patient and making masses social protection more intelligent. Taking the favored outlook of this technique, it achieves perfect security by putting something aside for the patient. Compared to traditional EHR systems, which use client-server architecture, the proposed system uses blockchain for improving efficiency and security. Blockchain technology addresses interoperability challenges and is based on open standards, provides a shared distributed view of the health data, and will also achieve widespread acceptance and deployment throughout all industries. Utilization of the proposed health blockchain that is described in this paper has the potential to engage millions of individuals, health care providers, health care entities, and medical researchers to share the vast amount of genetic, diet, lifestyle, environmental, and health data with a guaranteed security and privacy protection. Future research opportunities lie in a deeper analysis of the business processes in the manufacturing industry to further exploit the advantages of blockchain technology.

\section{REFERENCES}

[1] G. Jetley and H. Zhang, "Electronic health records in IS research: Quality issues, essential thresholds and remedial actions," Decis. Support Syst., pp. 113-137, 2019

[2] K. Wisner, A. Lyndon, and C. A. Chesla, "The electronic health record's impact on nurses' cognitive work: An integrative review," Int. J. Nurs. Stud., vol. 94, pp. 74-84, 2019.

[3] M. Hochman, "Electronic Health Records: a "Quadruple Win,' a "Quadruple Failure," or Simply Time for a Reboot?," J. Gen. Intern. Med., vol. 33, no. 4, pp. 397-399, Apr. 2018.

[4] Q. Gan, "Adoption of Electronic Health Record System : Multiple Theoretical Perspectives," 2014 47th Hawaii Int. Conf. Syst. Sci., pp. 2716-2724, 2014.

[5] Vehko et al., "Experienced time pressure and stress: electronic health records usability and information technology competence play a role," BMC Med. Inform. Decis. Mak., vol. 19, no. 1, p. 160, Aug. 2019.

[6] M. Reisman, "EHRs: The Challenge of Making Electronic Data Usable and Interoperable.," P T, vol. 42, no. 9, pp. 572575, 2017.

[7] W. W. Koczkodaj, M. Mazurek, D. Strzałka, A. WolnyDominiak, and M. Woodbury-Smith, "Electronic Health Record Breaches as Social Indicators," Soc. Indic. Res., vol. 141, no. 2, pp. 861-871, 2019. [8] S. T. Argaw, N. E. Bempong, B. Eshaya-Chauvin, and A. Flahault, "The state of research on cyberattacks against hospitals and available best practice recommendations: A scoping review," BMC Med. Inform. Decis. Mak., vol. 19, no. 1, pp. 1-11, 2019.

[8] A. McLeod and D. Dolezel, "Cyber-analytics: Modeling factors associated with healthcare data breaches," Decis. Support Syst., vol. 108, pp. 57-68, 2018.
[9] L. Coventry and D. Branley, "Cybersecurity in healthcare: A narrative review of trends, threats and ways forward," Maturitas, vol. 113, pp. 48-52, 2018 